\title{
A novel XPM based pulse retiming system incorporating a fibre grating based parabolic pulse shaper
}

\author{
F. Parmigiani, P. Petropoulos, M. Ibsen, M. A. F. Roelens, D. J. Richardson \\ Optoelectronics Research Centre, University of Southampton, SO17 1BJ, United Kingdom \\ Phone: +44(0)2380593136, FAX: +44(0)2380593142, e-mail: frp@orc.soton.ac.uk
}

\begin{abstract}
A$ novel all-optical, all-fibre pulse retiming system is experimentally demonstrated. Our technique relies on converting the timing jitter into frequency shift using XPM switching of the data signal with parabolic pulses shaped using a SSFBG.
\end{abstract}

\section{Introduction}

Timing jitter reduction (or pulse retiming) is likely to be an essential function within future high speed optical communication systems. A common approach to pulse retiming is the conversion of timing jitter into a linear chirp which can subsequently be removed after propagation of the signal in an optical fibre, or by reflection from a linearly chirped FBG. The required timing-to-frequency shift conversion step has previously been achieved either by using a phase modulator synchronously driven at the data repetition rate [1], or by optically switching the data pulses with synchronous, temporally square and linearly chirped broad pulses [2].

In this paper we present a demonstration of an alloptical analogue of the phase modulator-based retiming technique. Our scheme is based on CrossPhase Modulation (XPM) in a highly nonlinear fibre (HNLF) and utilizes the time derivative effect of XPMinduced spectral broadening to induce chirp onto the data pulses [3]. Pulses overlapping with the leading (trailing) edge of the control pulses experience a red (blue) shift of their spectrum due to XPM. The signal is then retimed on a bit-by-bit basis by propagating in an appropriate length of dispersive fibre, due to the varying group delay experienced by the different wavelengths. In order to ensure that the chirp applied on the mistimed pulses is linear over an extended range with respect to temporal offset from the nominal pulse centre the control pulses should have a parabolic shape, and be much broader than the data.

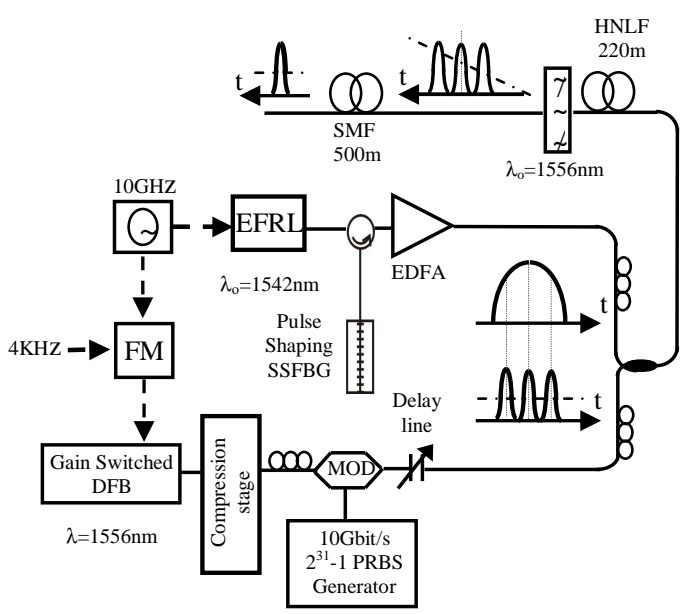

Figure 1: Experimental set-up and principle of the retiming scheme.
We generate parabolic pulses in our system in a linear and highly stable manner using pulse shaping in a superstructured fibre Bragg grating (SSFBG). We demonstrate this technique at $10 \mathrm{~Gb} / \mathrm{s}$, however since it is all-optical and relies on fibre nonlinearities, it should readily be scalable to repetition rates exceeding $320 \mathrm{~Gb} / \mathrm{s}$.

\section{Principle of operation and experimental set-up} The experimental set-up of the retiming scheme presented here is shown in Fig. 1. A mode-locked erbium fibre ring laser (EFRL) was used to generate $\sim 2$ ps soliton pulses at a repetition rate of $10 \mathrm{GHz}$. at $1542 \mathrm{~nm}$. These pulses were used as the input to the parabolic pulse shaper formed by a SSFBG. The shaped pulses were designed to have a parabolic envelope (FWHM of $\sim 10 \mathrm{ps}$ ) superimposed upon a $5^{\text {th }}$ order super-Gaussian profile which was used to smoothen the pulse edges and reduce its spectral extent. The grating was designed and fabricated with an appropriate phase and amplitude response to convert the 2 ps solitons into the target parabolic pulses. We characterised the pulses reflected off the SSFBG using second harmonic generation frequency-resolved optical gating (SHG-FROG) and calculated the time derivative of their intensity profile. We found this derivative to exhibit a good linear slope across the $10 \mathrm{ps}$ pulsewidth (Fig.2). Excellent agreement between the measured and calculated spectrum is achieved (Fig. 2 inset).

The data source used in our experiments was a gainswitched Distributed Feedback (DFB) laser, operating at $1556 \mathrm{~nm}$. These pulses were synchronised to the

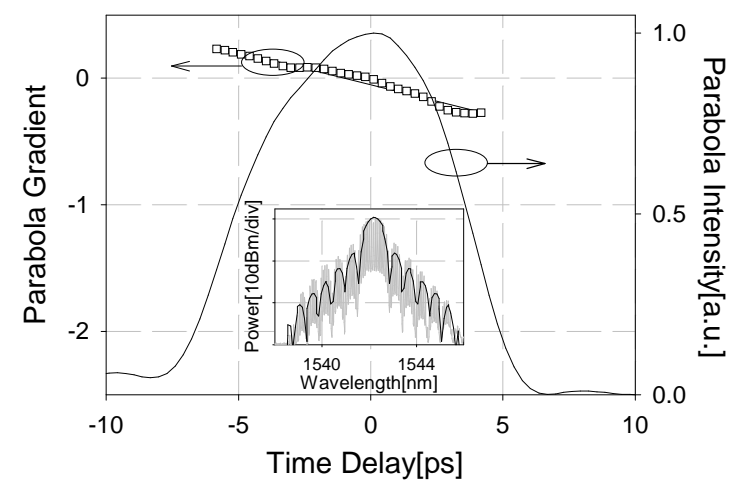

Figure 2: Intensity of the parabolic pulses measured using SHGFROG and its correspondent gradient. Inset: experimental (dashed line) and theoretical (solid line) spectra of the parabolic pulses. 
EFRL signal. We were able to artificially introduce timing jitter to the pulses by frequency modulating the $10 \mathrm{GHz}$ RF drive signal of the laser with a $4 \mathrm{kHz}$ tone. By varying the amplitude of the frequency modulation, we were able to control the amount of the induced timing jitter. The pulses were compressed down to 2ps using a fibre-based compressor. The pulses were then modulated by a $2^{31}-1$ pseudorandom bit sequence using a lithium niobate modulator and fed onto a $220 \mathrm{~m}$ of HNLF with a zero-dispersion wavelength at $1550 \mathrm{~nm}$, which ensured minimum walk-off time between the two signals. The average power of the control signal was $\sim 21 \mathrm{dBm}$, while the power of the data signal was kept quite low to avoid any spectral broadening due to self-phase modulation which would induce additional chirp. An optical delay line was used to temporally overlap the two signals. The data signal experienced XPM during propagation in the HNLF, acquiring a red frequency shift if the data pulse coincided with the leading edge of the much broader parabolic pulse or a blue shift if it coincided with the trailing edge of the parabolic pulse. The data signal then passed through a $5 \mathrm{~nm}$ broadband optical filter in order to filter out the control without shaping its spectrum, and was launched onto an appropriate length of single mode fiber $(500 \mathrm{~m})$ which was used to remove the chirp and retime the pulses.

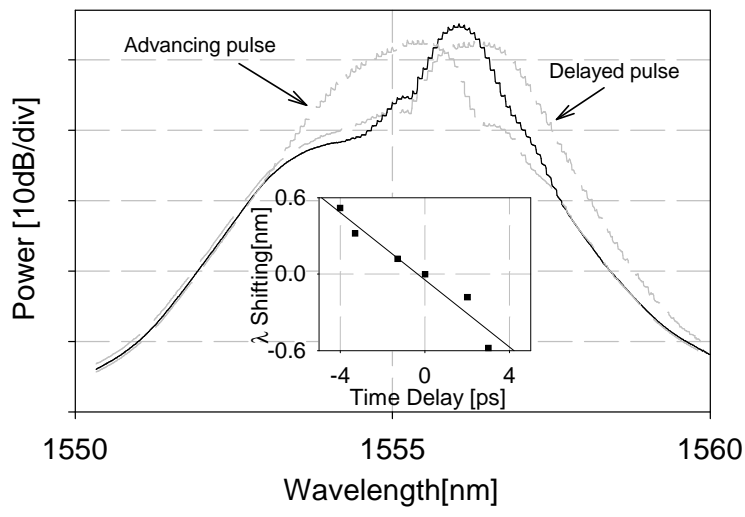

Figure 3: XPM spectra for different cases of overlapping between control and data signal. Inset: Wavelength shift versus timing shift.

\section{Results}

In order to characterize the performance of our timing-to-frequency shift conversion scheme, we first measured the wavelength shift of the XPM signal as a function of the time delay of the data relative to the centre of the parabolic pulses. Fig. 3 shows spectral traces of two cases of the data pulses overlapping with the leading (red shifting) or the trailing edge (blue shifting) of the control signal, and compares these to the case when no XPM was induced on the data signal at all. The inset figure shows a diagram of wavelength shift of the XPM signal as a function of the timing shift, confirming a linear relation between the two.

We evaluated the retiming of our scheme for several values of induced timing jitter by observing the eye diagrams at the input and output of the system on a digital communications analyser (DCA), and measuring the timing jitter distributions. These measurements are summarised in Fig.4, which demonstrates that with this system it was possible to suppress timing jitter in the incoming data stream of as much as $\sim 4 \mathrm{ps}$ (rms value), corresponding to a peak-to-peak ( $p-p)$ value of $\sim 18.2$ ps. Fig.4a-b show examples of the eye diagrams obtained for an incoming signal which had an rms timing jitter of $\sim 2.3 \mathrm{ps}$ and a $\mathrm{p}-\mathrm{p}$ value of $\sim 11.3 \mathrm{ps}$. The jitter of the pulses at the output of the retiming scheme was measured as $\sim 1 \mathrm{ps}$ (rms value), which corresponds to the noise limit of the DCA we used for our measurements.

Note that similar to other timing-to-frequency shift conversion techniques, an additional switching stage should be included at the output of the system in order to remove the wavelength shift from the switched signal, if the pulses are to be transmitted further. Finally, it is also worth noting that this technique is directly applicable to the simultaneous retiming of several synchronised WDM channels.

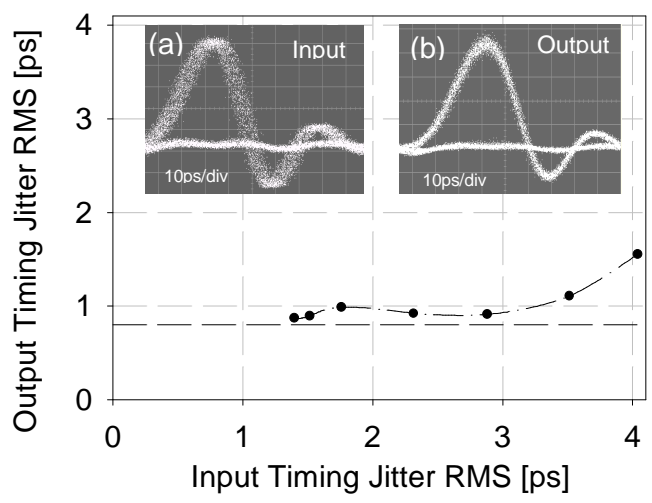

Figure 4: Output versus input timing jitter. Inset: eye diagrams (a) before and (b) after the retiming scheme for a 2.2ps input.

\section{Conclusions}

In conclusion, we have demonstrated a novel technique for the retiming of short optical pulses. The technique relies on timing-to-frequency shift conversion using XPM-induced spectral broadening in a HNLF. To ensure a linear chirp on the switched pulses, a parabolic pulse shape was used as the control to the HNLF, which was generated using pulse shaping in a SSFBG. The amount of jitter that can be compensated for is determined by the width of the reshaped pulses.

\section{References}

1 L. A. Jiang et al, Optics. Lett. 28 (2003), 78-80.

2 J. A. Harrison et al, Opt. Commun. 240 (2004), 221-226.

3 B. E. Olsson et al, IEEE Photon. Technol. Lett., 13 (2001), 875-877. 